\title{
JUILLET 1974
}

56॰ ANNÉE - N॰ 667

\section{revue internationale de la croix-rouge}

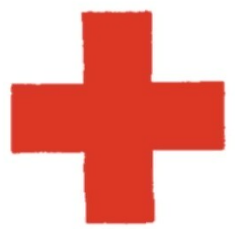

INTER ARMA CARITAS

GENËVE

COMITÉ INTERNATIONAL DE LA CROIX-ROUGE FONDÉ EN 1863 


\section{COMITÉ INTERNATIONAL DE LA CROIX-ROUGE}

MM. ERIC MARTIN, docteur en médecine, professeur honoraire de l'Université, Genève, président (membre depuis 1973)

JEAN PICTET, docteur en droit, président de la Commission juridique, vice-président (1967)

HARALD HUBER, docteur en droit, juge fédéral, vice-président (1969)

HANS BACHMANN, docteur en droit, directeur des finances de la ville, Winterthour (1958)

MM. MAX PETITPIERRE, docteur en droit, ancien conseiller fédéral (1961)

ADOLPHE GRAEDEL, ancien député au Conseil national. ancien secrétaire général de la Fédération internationale des ouvriers sur métaux (1965)

$M^{\text {mo }}$ DENISE BINDSCHEDLER-ROBERT, docteur en droit, professeur à l'Institut universitaire de hautes études internationales, Genève (1967)

MM. MARCEL A. NAVILlE, licencié ès lettres, président du CICR de 1969 à 1973 (1967)

JACQUES F. DE ROUGEMONT, docteur en médecine (1967)

ROGER GALLOPIN, docteur en droit, ancien directeur général du CICR (1967)

WALDEMAR JUCKER, docteur en droit, secrétaire de l'Union syndicale suisse (1967)

VICTOR H. UMBRICHT, docteur en droit, administrateur (1970)

PIERRE MICHELl, licencié en droit, ancien ambassadeur (1971)

GILBERT ETIENNE, professeur à l'Institut universitaire de hautes études internationales et à l'Institut d'études du développement, Genève (1973)

ULRICH MIDDENDORP, docteur en médecine, chef de la clinique chirurgicale de l'Hôpital cantonal, Winterthour (1973)

M"le MARION ROTHENBACH, diplômée M.S.W. de 1'Université de Michigan, maítre-assistant à l'Ecole des sciences sociales et politiques de l'Université, Lausanne (1973)

MM. HANS PETER TSCHUDI, docteur en droit, ancien conseiller fédéral (1973)

HENRY HUGUENIN, directeur de banque (1974)

GOTTFRIED DE SMIT, administrateur (1974)

Membres honoraires: M. JACQUES CHENEVIÈRE, vice-président d'honneur; M"1e LUCIE ODIER, vice-présidente d'honneur; MM. GUILlAUME BORDIER, PAUL CARRY, $M^{m}$ MARGUERITE GAUTIER-VAN BERCHEM, MM. SAMUEL A. GONARD, EDOUARD DE HALLER, RODOLFO OLGIATI, PAUL RUEGGER, DIETRICH SCHINDLER, FRÉDÉRIC SIORDET, ALFREDO VANNOTTI, ADOLF VISCHER.

\section{CONSEIL EXÉCUTIF}

M. ROGER GALLOPIN, président

M. VICTOR H. UMBRICHT, vice-président

$M^{\text {me }}$ DENISE BINDSCHEDLER-ROBERT

M. GILBERT ETIENNE

$D^{r}$ ULRICH MIDDENDORP

M. JEAN PICTET

M. GOTTFRIED DE SMIT

M. PIERRE MICHELI, membre suppleant 


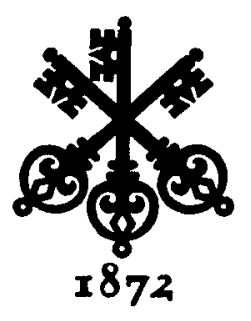

PRÉSENTE SUR LES CINQ CONTINENTS,

LA GRANDE BANQUE AUX TROIS CLEFS

EST À VOTRE DISPOSITION POUR EFFECTUER

TOUTES VOS OPÉRATIONS FINANCIĖRES

\section{SOCIÉTÉ DE BANQUE SUISSE}

SCHWEIZERISCHER BANKVEREIN - SOCIETA DI BANCA SVIZZERA

SWISS BANK CORPORATION

SIËGE DE GENEVE

2, RUE DE LA CONFÉDÉRATION

TÉL. 201111

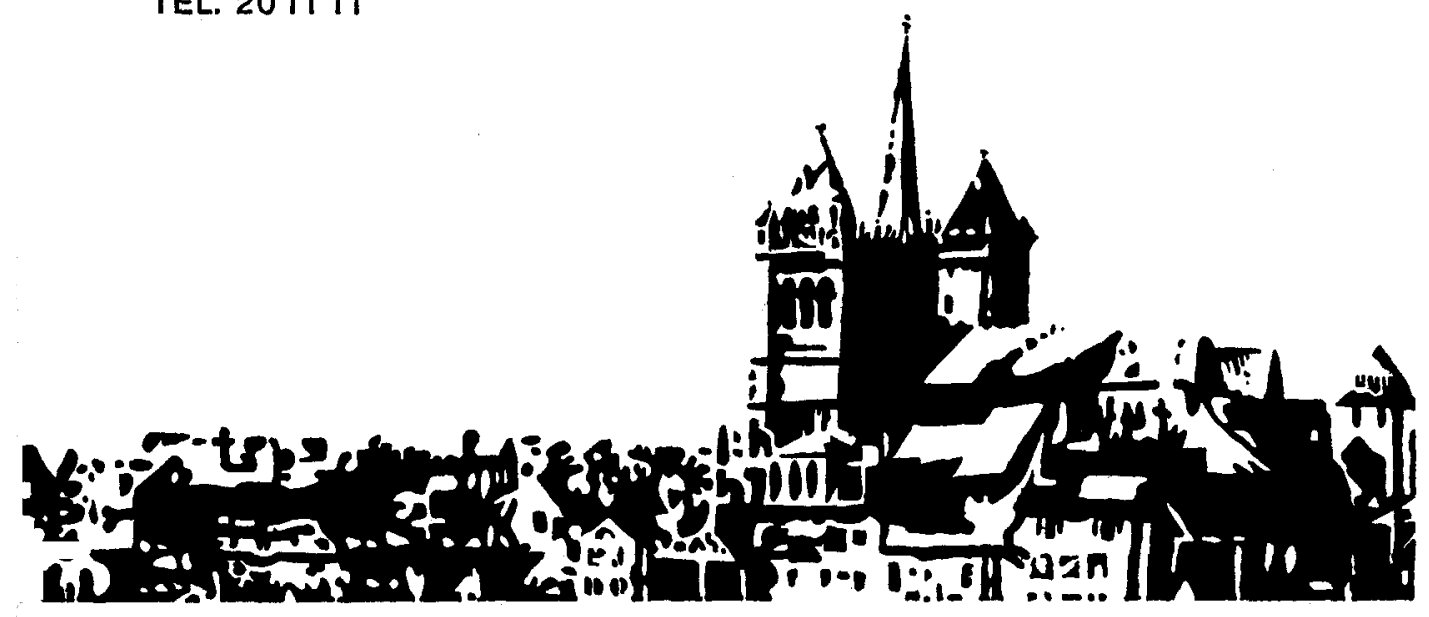




\section{SOCIETE FIDUCIAIRE ROMANDE OFOR S.A.}

Place Saint-Gervais 1, Genève - Tél. 317050

Expertises, revisions et organisations comptables

Domiciliation et administration de sociétés

Assainissements et liquidations

- Interventions et conseils en matière fiscale

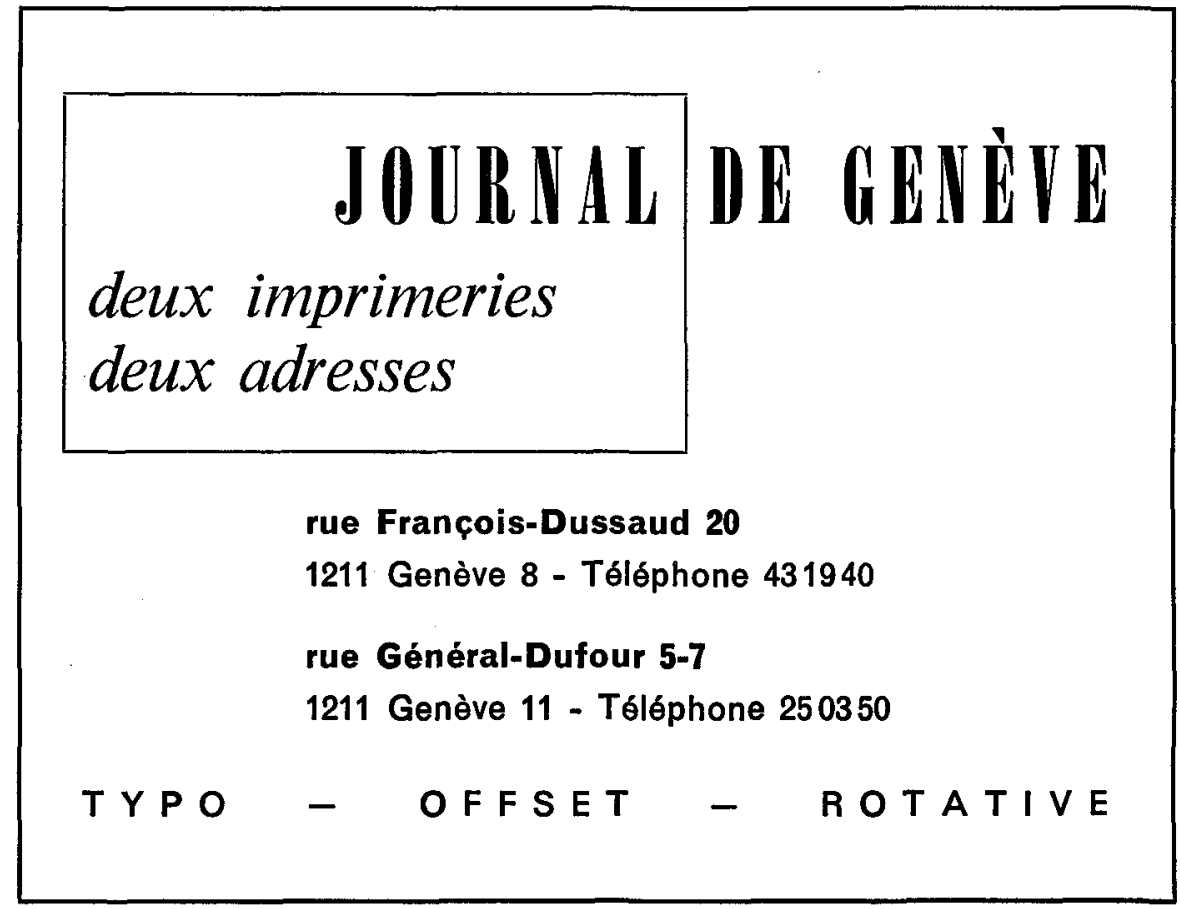




\section{REVUE INTERNATIONALE DE LA CROIX-ROUGE}

\section{SOMMAIRE}

COMITE INTERNATIONAL DE LA CROIX-ROUGE

DANS LE MONDE DE LA CROIX-ROUGE

LIVRES ET REVUES
JUILLET 1974 - №667

Z. S. Hantchef : La Croix-Rouge et les nouveaux aspects de la transfusion sanguine . . . . . 385

Une Convention peu connue sur le droit de la guerre 393

Rapatriement de prisonniers de guerre au Moyen-

Orient . . . . . . . . . . . . . . 405

Activités extérieures:

Rhodésie - République du Vietnam - Sous-continent asiatique - Europe - Moyen-Orient . . . 413

A Genève:

Le Roi et la Reine des Belges au CICR . . . . 416

Les hôtes du CICR . . . . . . . . . . . . . 416

Un nouveau film du CICR . . . . . . . . . . 419

Un appel du CICR et de la Ligue . . . . . . 420

Après la Journée mondiale de la Croix-Rouge . 422

Indochine . . . . . . . . . . . . . . 423 


\section{INTERNATIONAL REVIEW OF THE RED CROSS}

\section{SUPPLEMENTS}

DE LA REVUE

EN LANGUE ESPAGNOLE

EN LANGUE ALLEMANDE

REVUE INTERNATIONALE DE LA CROIX-ROUGE
Une édition en langue anglaise paraitt chaque mois. Elle est en principe identique à l'édition française, et peut être obtenue aux mêmes conditions.

Conferencia de expertos gubernamentales - En el Subcontinente Asiático 250000 personas repatriadas Difusión de los Convenios de Ginebra - Instituto Henry-Dunant - Un llamamiento del CICR y de la Liga.

Auf dem asiatischen Subkontinent - Verbreitung der Genfer Abkommen - Des IKRK beruft eine Regierungsexpertenkonferenz über die Waffen ein - Veröffentlichungen in deutscher Sprache - Henry-DunantInstitut - VI. regionale Tagung der Rotkreuzgesellschaften in Tegucigalpa.

Elle est publiée chaque mois par le Comité international de la Croix-Rouge.

17, avenue de la Paix, 1211 Genève (Suisse) - Compte de chèques postaux $12-1767$.

Abonnement un an: Fr. 30,-; le numéro: Fr. 3,-.

RÉDACTION : J.-G. LOSSIER

Seuls les textes signés par le Comité international de la Croix-Rouge engagent la responsabilité de celui-ci. 